\title{
ON EXPONENTS OF HOMOLOGY AND COHOMOLOGY OF FINITE GROUPS
}

\author{
JON F. CARLSON
}

(Communicated by Bhama Srinivasan)

\begin{abstract}
Let $G$ be a finite group and let $r$ be the maximum of the $p$-ranks of $G$ for all primes $p$ dividing the order to $G$. There exist positive integers $m$ and $n$ such that the exponents of $H^{n}(G, \mathbf{Z})$ and $H_{m}(G, \mathbf{Z})$ are greater than $|G|^{1 / r}$.
\end{abstract}

Suppose that $G$ is a finite group. It has long been known that the exponent of $H^{n}(G, \mathbf{Z})$ divides $|G|$, the order of $G$, for all $n>0$. Beyond this fact very little has been established concerning exponents of cohomology groups, and what is known seems to be mostly about upper bounds for the exponents. Lewis [3] found an element of order $p^{2}$ in $H^{2 p}(G, \mathbf{Z})$ when $G$ is the group of order $p^{3}$ and exponent $p$ ( $p$ odd). The purpose of this paper is to give a general lower bound for exponents on group homology and cohomology. In some sense it explains the phenomenon observed by Lewis.

Throughout let $R$ denote either the ordinary integers $\mathbf{Z}$ or the localization $\mathbf{Z}_{p}$ of $\mathbf{Z}$ at a prime $p$. Define the $R$-rank of $G$ to be equal to the $p$-rank of $G$ if $R=\mathbf{Z}_{p}$ and equal to the maximum of the $p$-ranks of $G$ for all primes $p$ if $R=\mathbf{Z}$. The main result is the following.

THEOREM 1. Let $r$ be the $R$-rank of $G$. There exist an infinite number of positive integers $m$ and $n$ such that

$$
\exp H^{n}(G, R) \geq|R /(g)|^{1 / r} \quad \text { and } \quad \exp H^{m}(G, R) \geq|R /(g)|^{1 / r},
$$

where $|R /(g)|$ is the order of the group $R /(g), g=|G|$.

In the case $R=\mathbf{Z},|R /(g)|=|G|$. If $R=\mathbf{Z}_{p}$ and $|G|=p^{a} \cdot q$, then $R /(g)$ has order $p^{a}$. It is well known that, because $\mathbf{Z}_{p}$ is a flat $\mathbf{Z}$-module, $H^{n}(G, \mathbf{Z})=\bigoplus_{p} H^{n}\left(G, \mathbf{Z}_{p}\right)$ and $H_{m}(G, \mathbf{Z})=\bigoplus_{p} H_{m}\left(G, \mathbf{Z}_{p}\right)$ for $m, n \geq 1$. That is, $H^{n}\left(G, \mathbf{Z}_{p}\right)$ is isomorphic to $H^{n}(G, \mathbf{Z})_{p}$, the $p$-primary part of $H^{n}(G, \mathbf{Z})$. Hence in case $R=\mathbf{Z}_{p}$ the theorem implies the following.

COROLlaRY 2. Suppose that $|G|=p^{a} \cdot q,(p, q)=1$, and $a \geq 1$. Let $r$ be the $p$-rank of $G$, and let $s$ be the least integer that is greater than or equal to a/r. Then there exist an infinite number of positive integers $m$ and $n$ such that $p^{s}$ divides $\exp H^{n}(G, \mathbf{Z})$ and $\exp H_{m}(G, \mathbf{Z})$.

The proof of Theorem 1 is little more than a combination of two results. The first demonstrates that certain types of complexes of projective $R G$-modules can

Received by the editors December 8, 1986.

1980 Mathematics Subject Classification (1985 Revision). Primary 20J06.

Key words and phrases. Homology of finite groups, cohomology of finite groups.

This work was partly supported by the NSF. 
be constructed [1]. The theorem is then almost immediate from the second result [2]. For the sake of completeness we present a brief description of the construction. (See [1] for details and references.)

Let $(p)$ be a maximal prime ideal in $R$. For $\varsigma \in H^{n}(G, R)$, let $\bar{\zeta} \in H^{n}(G, R /(p))$ denote its reduction modulo $p$. Let $r$ be the $R$-rank of $G$ and choose homogeneous elements $\zeta_{1}, \ldots, \zeta_{r} \in H^{*}(G, R)$ such that, for every such prime ideal $(p)$, the elements $\bar{\zeta}_{1}, \ldots, \bar{\zeta}_{r}$ have the property that $H^{*}(G, R /(p)) /\left(\bar{\zeta}_{1}, \ldots, \bar{\zeta}_{r}\right)$ is a finitedimensional algebra over $R /(p)$. Also if $R=\mathbf{Z}$ and $r_{p}$ is the $p$-rank of $G$, then assume that $\left(\bar{\zeta}_{1}, \ldots, \bar{\zeta}_{r_{p}}\right)$ generate an ideal of finite codimension in $H^{*}(G, R /(p))$. If $V_{G}(R /(p))$ is the maximal ideal spectrum of $H^{*}(G, R /(p)) / \operatorname{Rad} H^{*}(G, R /(p))$ and if $V_{G}\left(\bar{\zeta}_{i}\right)$ is the subvariety of $V_{G}(R /(p))$ corresponding to the ideal generated by $\bar{\zeta}_{i}$, the preceding is equivalent to the statement that

$$
V_{G}\left(\bar{\zeta}_{1}\right) \cap \cdots \cap V_{G}\left(\bar{\zeta}_{r}\right)=\{0\} .
$$

Such sets of elements exist because $r_{p}$ is the Krull dimension of $H^{*}(G, R /(p))$.

Let

$$
\cdots \rightarrow F_{1} \rightarrow F_{0} \rightarrow R \rightarrow 0
$$

be an $R G$-projective resolution of $R$. We need two more assumptions, neither of which cause any problems as far as the existence of sets $\left\{\varsigma_{1}, \ldots, \zeta_{r}\right\}$ as above. First assume that $l_{i}=\operatorname{deg}\left(\varsigma_{i}\right)>1$. Also if $R=\mathbf{Z}$ we insist that the $p$-primary part of $\varsigma_{i}$ is zero if $i>r_{p}$, the $p$-rank of $G$. Then each $\zeta_{i}$ is represented by a cocycle $\hat{\zeta}_{i}: F_{l_{i}} \rightarrow R$ which is a surjection. Now consider the following commutative diagram:

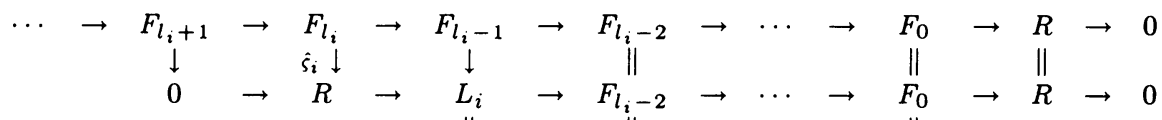

$$
\begin{aligned}
& 0 \rightarrow C_{l_{i-1}}^{\|} \rightarrow C_{l_{i-2}}^{(i)} \rightarrow \cdots \rightarrow C_{0}^{(i)} \rightarrow 0
\end{aligned}
$$

Here the middle row is the pushout of the top row along $\hat{\zeta}_{i}$. The bottom row is a complex which we denote by $C^{(i)}$. It is clear that $H_{j}\left(C^{(i)}\right)=0=H^{j}\left(C^{(i)}\right)$ if $j \neq 0$, $l_{i}-1$, while $\bar{H}_{j}\left(C^{(i)}\right) \cong R \cong H^{j}\left(C^{(i)}\right)$ if $j=0$ or $j=l_{i}-1$. From the conditions on $\varsigma_{1}, \ldots, \zeta_{r}$ it follows that $L=L_{1} \otimes \cdots \otimes L_{r}$ is a projective $R G$-module because $L /(p) L$ is a projective $(R /(p)) G$-module for all primes $p$. Now let $C=C\left(\varsigma_{1}, \ldots, \varsigma_{r}\right)$ be the total complex of the tensor product $C=C^{(1)} \otimes_{R} \cdots \otimes_{R} C^{(r)}$. In summary, using the Künneth tensor formula, we get the following.

THEOREM $3[\mathbf{1}]$. Let $r$ be the $R$-rank of $G$. There exist homogeneous elements $\varsigma_{1}, \ldots, \varsigma_{r} \in H^{*}(G, R)$ satisfying the following conditions.

(i) $H^{*}(G, R /(p)) /\left(\bar{\zeta}_{1}, \ldots, \bar{\zeta}_{r}\right)$ is a finite-dimensional algebra over $R /(p)$ for all maximal ideals $(p) \subseteq R$.

(ii) $\operatorname{deg} \zeta_{i}=l_{i}>1$ for all $i$.

(iii) If $R=\mathbf{Z}$, then the $p$-primary part of $\varsigma_{i}$ is zero for $i>r_{p}$, the $p$-rank of $G$.

If $\varsigma_{1}, \ldots, \varsigma_{r}$ satisfy the above conditions, then $C=C\left(\varsigma_{1}, \ldots, \varsigma_{r}\right)$ is a complex of projective $R G$-modules such that every $H_{j}(C)$ and $H^{j}(C)$ is a direct sum of copies of $R$. In particular

$$
H^{j}(C)=\sum H^{j_{1}}\left(C^{(1)}\right) \otimes \cdots \otimes H^{j_{r}}\left(C^{(r)}\right)
$$


and

$$
H_{j}(C)=\sum H_{j_{1}}\left(C^{(1)}\right) \otimes \cdots \otimes H_{j_{r}}\left(C^{(r)}\right),
$$

the sum being over all $r$-tuples $\left(j_{1}, \ldots, j_{r}\right)$ such that $j_{1}+\cdots+j_{r}=j$.

The other fact that we require is the following. Browder's proof for the case $R=\mathbf{Z}$ is trivially generalized to the other situations.

THEOREM 4 [2]. Let $C$ be a finite nonnegative chain complex of projective $R G$-modules such that $H_{0}(C)=R$. Then as elements of $R$

$$
|G| \text { divides } \prod_{j \geq 1} \exp H^{j+1}\left(G, H_{j}(C)\right)
$$

and

$$
|G| \text { divides } \prod_{j \geq 1} \exp H_{j+1}\left(G, H^{j}(C)\right) \text {. }
$$

ProOF OF ThEOREM 1 . Suppose that $r$ is the $R$-rank of $G$, and that $\zeta_{1}, \ldots, \zeta_{r}$ satisfy all of the conditions necessary for Theorem 3 to hold. It may be observed that $\zeta_{1}^{n_{1}}, \ldots, \zeta_{r}^{n_{r}}$ also satisfies the same hypotheses for any positive integers $n_{1}, \ldots, n_{r}$. Also $\operatorname{deg}\left(\varsigma_{i}^{n_{i}}\right)=n_{i} \operatorname{deg}\left(\varsigma_{i}\right)$, so we may assume that $\varsigma_{1}, \ldots, \zeta_{r}$ all have the same degree $l$. Then the complex $C$ has homology and cohomology only in degrees $j(l-1)$ for $j=0,1, \ldots, r$. Consequently Theorem 4 implies that $|G|$ divides $\prod_{j=1}^{r} \exp H^{j(l-1)+1}(G, R)$ in $R$. Thus $\exp H^{n}(G, R) \geq|R /(g)|^{1 / r}$ for some $j$, where $n=j(l-1)+1$. This proves existence of one such $n$. To obtain another simply replace every $\zeta_{i}$ by $\zeta_{i}^{t}$ for $t \cdot \operatorname{deg} \zeta_{i}>n$ and repeat the process. The proof for homology is almost the same.

For cohomology the existence of one value of $n$ automatically implies the existence of an infinite number, because the ring $H^{*}(G, R)$ has elements of positive degree that are not divisors of zero.

\section{REFERENCES}

1. D. J. Benson and J. F. Carlson, Complexity and multiple complexes, Math. Z. 195 (1987), 221-238.

2. W. Browder, Cohomology and group actions, Invent. Math. 71 (1983), 599-607.

3. G. Lewis, The integral cohomology rings of groups of order $p^{3}$, Trans. Amer. Math. Soc. 132 (1968), 501-529.

Department of Mathematics, University of Georgia, Athens, Georgia 30602 